\title{
CONVERGENCE OF PETVIASHVILI'S ITERATION METHOD FOR NUMERICAL APPROXIMATION OF STATIONARY SOLUTIONS OF NONLINEAR WAVE EQUATIONS*
}

\author{
DMITRY E. PELINOVSKY ${ }^{\dagger}$ AND YURY A. STEPANYANTS ${ }^{\ddagger}$ \\ In memory of Vladimir I. Petviashvili (1936-1993), who made an outstanding contribution to the \\ theory of nonlinear waves. \\ Abstract. We analyze a heuristic numerical method suggested by V.I. Petviashvili in 1976 for \\ approximation of stationary solutions of nonlinear wave equations. The method is used to construct \\ numerically the solitary wave solutions, such as solitons, lumps, and vortices, in a space of one \\ and higher dimensions. Assuming that the stationary solution exists, we find conditions when the \\ iteration method converges to the stationary solution and when the rate of convergence is the fastest. \\ The theory is illustrated with examples of physical interest such as generalized Korteweg-de Vries, \\ Benjamin-Ono, Zakharov-Kuznetsov, Kadomtsev-Petviashvili, and Klein-Gordon equations.
}

Key words. nonlinear evolution equations, solitary waves, numerical approximations, iteration methods, convergence and stability, linearized operators

AMS subject classifications. 65N35, 35Q51, 35Q53, 35P20

DOI. $10.1137 / \mathrm{S} 0036142902414232$

1. Introduction. Nonlinear waves and vortices are often described by partial differential equations, whose solutions cannot be found analytically even in a space of one dimension. Numerical computations are used to approximate various solutions, including stationary solutions. An effective numerical method for computing solitary wave solutions in a space of two dimensions was proposed by V. I. Petviashvili in the context of the Kadomtsev-Petviashvili equation with positive dispersion (KPI equation) [P76]. The numerical method was shown to converge to a stationary solution, but no analysis or proof was given. One year later, the very same solution was found analytically [MZ77], referred to as the two-dimensional soliton or lump. After the pioneering work [P76], Petviashili's numerical method was applied to numerous nonlinear problems in modern mathematical physics [PP92].

In this paper, we prove the convergence theorem for Petviashvili's numerical method in a context of a nonlinear scalar wave equation with power nonlinearity. We assume that the stationary solution exists in a suitable function space, when the method is well defined. The method clearly diverges in the cases when no stationary solution exists in such spaces. We derive conditions on parameters of the numerical method and on the spectrum of a linearized operator associated with the stationary solution, when the method converges to the stationary solution.

We start with a nonlinear scalar wave equation with power nonlinearity in one dimension:

$$
u_{t}-(\mathcal{L} u)_{x}+p u^{p-1} u_{x}=0,
$$

where $u: \mathbb{R} \times \mathbb{R}_{+} \mapsto \mathbb{R}, p>1$, and $\mathcal{L}$ is a linear self-adjoint nonnegative pseudodiffer-

* Received by the editors September 9, 2002; accepted for publication (in revised form) November 12, 2003; published electronically September 18, 2004.

http://www.siam.org/journals/sinum/42-3/41423.html

${ }^{\dagger}$ Department of Mathematics, McMaster University, Hamilton, ON, Canada, L8S 4K1 (dmpeli@

math.mcmaster.ca). The work of this author was supported by NSERC grant 5-36694.

${ }^{\ddagger}$ Environment at ANSTO, PMB 1, Menai, NSW, 2234, Australia (ysx@ansto.gov.au). 
ential operator in $x$ with constant coefficients, such that

$$
\langle u, \mathcal{L} u\rangle=\langle\mathcal{L} u, u\rangle \geq 0, \quad\langle f, g\rangle=\int_{-\infty}^{\infty} \bar{f}(x) g(x) d x .
$$

Stationary solutions of (1.1) are of the form $u(x, t)=\Phi(x-c t)$, where $c$ is an eigenvalue and $\Phi(x)$ is a bound state of the boundary-value problem on $x \in \mathbb{R}$,

$$
c \Phi+\mathcal{L} \Phi=\Phi^{p},
$$

such that $\lim _{|x| \rightarrow \infty} \Phi(x)=0$. The paramater $c$, which is typically continuous, has a physical meaning of a speed of the stationary wave. The bound state $\Phi(x)$ belongs to the function space $X(\mathbb{R})$, defined in Assumption 1.1.

We employ the Fourier transform,

$$
u(x)=\frac{1}{2 \pi} \int_{-\infty}^{\infty} \hat{u}(k) e^{i k x} d k, \quad \hat{u}(k)=\int_{-\infty}^{\infty} u(x) e^{-i k x} d x
$$

and rewrite the boundary-value problem (1.3) in the form

$$
[c+v(k)] \hat{\Phi}(k)=\widehat{\Phi^{p}}(k),
$$

where $v(k)$ is the range of $\mathcal{L}$ in the Fourier space. If $\mathcal{L}$ is a nonnegative pseudodifferential operator of order $m$, the function $v(k)$ is an $m$ th order polynomial of $|k|$, such that $v(k) \geq 0$. The function $v(k)$ has meaning of phase velocity of linear waves (infinitesimal perturbations) of the scalar wave equation (1.1). Resonance between nonlinear bound states and linear waves is excluded if $c+v(k) \neq 0$ for any $k \in \mathbb{R}$. For the nonnegative operator $\mathcal{L}$ with $v(k) \geq 0$, the resonance is excluded for $c>0$.

Assumption 1.1. Let $m$ be the order of a linear pseudodifferential operator $\mathcal{L}$, $p>1, v(k) \geq 0$, and $c>0$. There exists a real analytical solution of the boundaryvalue problem (1.5) in the function space

$$
X=L^{2}(\mathbb{R}) \cap L^{p+1}(\mathbb{R}) \cap H^{m / 2}(\mathbb{R}) .
$$

A naive iterative algorithm for numerical approximation of $\hat{\Phi}(k)$ in the problem (1.5) can be proposed in the form

$$
\widehat{u}_{n+1}(k)=\frac{\widehat{u_{n}^{p}}(k)}{c+v(k)},
$$

where $\widehat{u}_{n}(k)$ is the Fourier transform of $u_{n}(x)$ and $u_{n}(x)$ is the $n$th iteration of the numerical solution. However, this algorithm usually diverges, even if a fixed point $\hat{\Phi}(k)$ exists in the nonlinear problem (1.5). A modified iterative procedure is proposed by introducing the stabilizing factor $M_{n}[\mathrm{P} 76]$,

$$
\widehat{u}_{n+1}(k)=M_{n}^{\gamma} \frac{\widehat{u_{n}^{p}}(k)}{c+v(k)},
$$

where the stabilizing factor $M_{n}$ is computed as

$$
M_{n}=M_{n}\left[\widehat{u}_{n}\right]=\frac{\int_{-\infty}^{\infty}[c+v(k)]\left[\widehat{u}_{n}(k)\right]^{2} d k}{\int_{-\infty}^{\infty} \widehat{u}_{n}(k) \widehat{u_{n}^{p}}(k) d k},
$$


and $\gamma$ is a free parameter, which must be chosen for convergence of the sequence $\left\{u_{n}(x)\right\}_{n=0}^{\infty}$. The fixed points of the iterative map (1.8)-(1.9) are the same as the bound states $\hat{\Phi}(k)$ of the nonlinear boundary-value problem (1.5).

LEMma 1.2. A set of fixed points of the iteration map (1.8)-(1.9) coincides with a set of bound states $\hat{\Phi}(k)$ of the boundary-value problem (1.5), provided that $\gamma \neq 1+2 n$, $n \in \mathbb{Z}$.

Proof. If $\widehat{u}_{n}(k)=\hat{\Phi}(k)$ is a solution of the boundary-value problem (1.5), then $M_{n}=1$ from (1.9) and $\widehat{u}_{n+1}(k)=\hat{\Phi}(k)$ from (1.8). Therefore, the solution $\hat{\Phi}(k)$ is a fixed point of the iteration map (1.8)-(1.9). In the other direction, let $\widehat{u}_{*}(k)$ be a fixed point of the iteration map (1.8)-(1.9). Multiplying (1.8) by $[c+v(k)] \widehat{u}_{*}(k)$ and integrating over $k$, we find $M_{*}=M_{*}^{\gamma}$. When $\gamma \neq 1+2 n, n \in \mathbb{Z}$, there exist only two solutions: $M_{*}=0$ or $M_{*}=1$. Since $c+v(k)>0$ for any $k \in \mathbb{R}$, the former solution is equivalent to a trivial zero fixed point: $\widehat{u}_{*}(k)=0$. The fixed point of (1.8) with $M_{*}=1$ satisfies the boundary-value problem (1.5), such that $\widehat{u}_{*}(k)=\hat{\Phi}(k)$.

When $\gamma=0$, the iterative method (1.8) is the same as in (1.7) and it diverges in most cases as was mentioned above. Nevertheless, a nonempty range for $\gamma$ can be found empirically, when the method converges to the bound state $\Phi(x)$, starting with $u_{0} \in X(\mathbb{R})$ such that $u_{n} \in X(\mathbb{R}), \lim _{n \rightarrow \infty} u_{n}(x)=\Phi(x)$, and $\lim _{n \rightarrow \infty} M_{n}=1$. For $p=2$ (quadratic nonlinearity), Petviashvili has found empirically that the iteration method (1.8)-(1.9) converges for $1<\gamma<3$, with the fastest rate of convergence at $\gamma=2$ [PP92]. He also noticed that the fastest rate of convergence occurs when the degree of the uniformity of the right-hand side of (1.8) is zero with respect to $\widehat{u}_{n}(k)$. The convergence results do not depend on the actual dependence $v(k)$, provided that $c+v(k)>0$ [PP92].

In this paper, we prove that the iteration method (1.8)-(1.9) converges for $1<$ $\gamma<(p+1) /(p-1)$ under some additional assumptions on the spectrum of a linearized operator associated with the bound state $\Phi(x)$. The fastest rate of convergence occurs for $\gamma=\gamma_{*}=p /(p-1)$.

From a practical point, the iteration procedure can be stopped when $\left|M_{n}-1\right| \leq \varepsilon$ for any given small $\varepsilon>0$. Therefore, parameter $\varepsilon$ defines the distance between $u_{n}(x)$ and $\Phi(x)$ that measures the numerical error in the sense of the integrals in (1.9). Two additional sources of numerical errors come from the use of spectral methods, such as (i) the truncation of the integration domain $k \in \mathbb{R}$ by a finite interval $k \in[-K, K]$ and (ii) the discretization of the integrals at a finite number of grid points.

The paper is organized as follows. Section 2 describes properties of the linearized operator associated with the scalar wave equation (1.1) and also formulates the main convergence theorem. Section 3 presents the proof of the convergence theorem. Convergence of the special sequences, which are self-similar to the bound states, is considered in section 4. Examples of the iteration method (1.8)-(1.9) in one and two dimensions are studied in sections 5 and 6.

2. Spectral properties of the linearized operator. Here we study properties of the linearized operator associated with the nonlinear wave equation (1.1) at $u=$ $\Phi(x-c t)$

$$
\mathcal{H}=c+\mathcal{L}-p \Phi^{p-1}(x),
$$

such that $\mathcal{H}: L^{2}(\mathbb{R}) \rightarrow L^{2}(\mathbb{R})$ and $\langle f, \mathcal{H} g\rangle=\langle\mathcal{H} f, g\rangle$. Since the operator $\mathcal{H}$ is selfadjoint in $L^{2}(\mathbb{R})$, its spectrum is real, eigenvalues of the discrete spectrum have equal geometric and algebraic multiplicities, and the spectral decomposition of $L^{2}(\mathbb{R})$ is orthogonal. 
The continuous spectrum of $\mathcal{H}$ is positive and bounded away from zero under Assumption 1.1. The null-space of $\mathcal{H}$ is not empty and includes at least one eigenfunction: $\mathcal{H} \Phi^{\prime}(x)=0$, since the nonlinear equation (1.1) has the translation symmetry: $u(x, t) \rightarrow u\left(x-x_{0}, t\right)$. The negative spectrum of $\mathcal{H}$ is not empty, since $\mathcal{H} \Phi(x)=(1-p) \Phi^{p}(x)$ and

$$
\begin{aligned}
\langle\mathcal{H} \Phi, \Phi\rangle=-(p-1)\left\langle\Phi^{p}, \Phi\right\rangle & =-\frac{(p-1)}{2 \pi} \int_{-\infty}^{\infty} \widehat{\Phi}(k) \widehat{\Phi^{p}}(k) d k \\
& =-\frac{(p-1)}{2 \pi} \int_{-\infty}^{\infty}[c+v(k)][\hat{\Phi}(k)]^{2} d k<0 .
\end{aligned}
$$

The analysis does not depend on the number and type of positive eigenvalues of $\mathcal{H}$. We summarize the main properties of the spectrum of $\mathcal{H}$ in the following assumption.

Assumption 2.1. The spectrum of $\mathcal{H}$ in $L^{2}(\mathbb{R})$ consists of eigenvalues $\mu$ of the discrete spectrum for $\mu<c$ and the continuous spectrum for $\mu \geq c$. The null-space of $\mathcal{H}$ is one dimensional with the eigenfunction $\Phi^{\prime}(x)$. The negative space of $\mathcal{H}$ has dimension $n(\mathcal{H}) \geq 1$.

Two linear eigenvalue problems are associated with the linearized operator $\mathcal{H}$ on $x \in \mathbb{R}:$

$$
\text { Problem I: } \quad \partial_{x} \mathcal{H} U=\lambda U
$$

and

$$
\text { Problem II: } \quad \mathcal{H} U=\lambda(c+\mathcal{L}) U .
$$

Problem I occurs in the linearization of the nonlinear wave equation (1.1) with a small perturbation to the bound state: $u=\Phi(x-c t)+U(x-c t) e^{\lambda t}$. The nonzero spectrum of $\partial_{x} \mathcal{H}$ is defined in the constrained function space $X_{c}(\mathbb{R})$,

$$
X_{c}=\left\{U \in L^{2}(\mathbb{R}):\langle\Phi, U\rangle=0\right\},
$$

since $\lambda\langle\Phi, U\rangle=\left\langle\Phi, \partial_{x} \mathcal{H} U\right\rangle=-\left\langle\mathcal{H} \Phi^{\prime}, U\right\rangle=0$. The spectrum of $\partial_{x} \mathcal{H}$ in $X_{c}(\mathbb{R})$ gives stability or instability of the bound state $\Phi(x)$ in the time evolution of the nonlinear wave equation (1.1). If there exists $\lambda \in \mathbb{C}$ such that $\operatorname{Re}(\lambda)>0$, the bound state is spectrally unstable and the perturbations grow exponentially in time. If the spectrum is located at the axis $\operatorname{Re}(\lambda)=0$, the bound state is weakly spectrally stable and the perturbation may grow at most as powers of time. The spectral stability-instability theorem for the scalar wave equation (1.1) can be formulated as follows.

TheOrem 2.2 ([BSS87, PW92]). . Let $P_{s}(c)=\langle\Phi, \Phi\rangle$ be a $C^{1}$ function of $c$ for $c>0$ and Assumptions 1.1 and 2.1 be satisfied. The bound state $\Phi(x)$ is weakly spectrally stable with respect to the time evolution problem $(2.3)$ if $n(\mathcal{H})=1$ and $P_{s}^{\prime}(c)>0$. The bound state $\Phi(x)$ is spectrally unstable if $n(\mathcal{H})=1$ and $P_{s}^{\prime}(c)<0$. The negative space of the operator $\mathcal{H}$ in the constrained function space $X_{c}(\mathbb{R})$ has the dimension $n(\mathcal{H})-1$ if $P_{s}^{\prime}(c)>0$ and the dimension $n(\mathcal{H})$ if $P_{s}^{\prime}(c)<0$.

Spectral stability of the bound state $\Phi(x)$ occurs if the negative space of $\mathcal{H}$ is empty in the constrained function space $X_{c}(\mathbb{R})$ and the spectral instability occurs if the negative space of $\mathcal{H}$ is one dimensional in $X_{c}(\mathbb{R})$. On the contrary, the convergence of the iteration method (1.8)-(1.9) does not depend on spectral stability or instability of bound states. Convergence of the iteration method is related to the spectrum of Problem II, which occurs in the linearization of the iteration method (1.8)-(1.9); see (3.5) below. 
We consider the spectrum of the operator $(c+\mathcal{L})^{-1} \mathcal{H}$ in a different constrained space $X_{p}(\mathbb{R})$,

$$
X_{p}=\left\{U \in L^{2}(\mathbb{R}):\left\langle\Phi^{p}, U\right\rangle=0\right\} .
$$

The spectrum of $(c+\mathcal{L})^{-1} \mathcal{H}$ diagonalizes simultaneously two linear operators: $\mathcal{H}$ and $(c+\mathcal{L})$. Since $(c+\mathcal{L})$ is positive, all eigenvalues $\lambda$ are real and the algebraic multiplicity of eigenvalues equals to their geometric multiplicity. Therefore, the spectral decomposition of $L^{2}(\mathbb{R})$ is orthogonal with respect to the positive weighted inner product $\langle U,(c+\mathcal{L}) U\rangle$. In particular, due to the constraint (2.6), the eigenfunction $U(x)$ is orthogonal with respect to $(c+\mathcal{L})$ to $\Phi(x)$, which is the eigenfunction of Problem II for $\lambda=1-p<0$.

Before formulating our main result (Theorem 2.8), we study the spectrum of $(c+\mathcal{L})^{-1} \mathcal{H}$ in $X_{p}(\mathbb{R})$ under Assumption 2.1. Our analysis appears similar to the Birman-Schwinger principle for Schrödinger operators in quantum mechanics [BS87].

Lemma 2.3. The negative space of $\mathcal{H}$ in $X_{p}(\mathbb{R})$ has the dimension $n(\mathcal{H})-1$.

Proof. The number of eigenvalues of $\mathcal{H}$ in the constrained function space $X_{p}(\mathbb{R})$ can be found from the constrained eigenvalue problem

$$
\mathcal{H} \psi=\mu \psi-\nu \Phi^{p}(x),
$$

where $(\mu, \psi)$ is the eigenvalue-eigenfunction pair of $\mathcal{H}$ in $X_{p}(\mathbb{R})$ and $\nu$ is the Lagrange multiplier defined from the constraint $\left\langle\Phi^{p}, \psi\right\rangle=0$. The operator $\mathcal{H}-\mu$ is invertible for any $\mu$ not in the spectrum of $\mathcal{H}$, where the spectral decomposition for $\psi(x)$ takes the form

$$
\psi(x)=\nu\left[\sum_{\mu_{k}<0} \frac{\left\langle u_{k}, \Phi^{p}\right\rangle}{\mu-\mu_{k}} u_{k}(x)+\sum_{\mu_{k}>0} \frac{\left\langle u_{k}, \Phi^{p}\right\rangle}{\mu-\mu_{k}} u_{k}(x)\right] .
$$

Here $\left(\mu_{k}, u_{k}\right)$ is the eigenvalue-eigenfunction pair of $\mathcal{H}$ in $L^{2}(\mathbb{R})$ and the formal sum $\sum_{\mu_{k}>0}$ includes also the integral over the positive continuous spectrum of $\mathcal{H}$. The set of eigenfunctions $\left\{u_{k}(x)\right\}_{k}$ is assumed to be orthogonal and normalized. The set of eigenvalues $\mu$ of $\mathcal{H}$ in $X_{p}(\mathbb{R})$ consists of two subsets. The first subset is given by eigenvalues $\mu_{k}$, whose eigenfunctions $u_{k}(x)$ belong to $X_{p}(\mathbb{R})$. The other subset is defined by zeros of the function

$$
F(\mu)=\frac{1}{\nu}\left\langle\Phi^{p}, \psi\right\rangle=\sum_{\mu_{k}<0} \frac{\left|\left\langle\Phi^{p}, u_{k}\right\rangle\right|^{2}}{\mu-\mu_{k}}+\sum_{\mu_{k}>0} \frac{\left|\left\langle\Phi^{p}, u_{k}\right\rangle\right|^{2}}{\mu-\mu_{k}} .
$$

We study zeros of $F(\mu)$ by direct application of the theory of constrained variational problems [P04]. The function $F(\mu)$ is monotonically decreasing for $\mu \leq 0$ and $\mu \neq \mu_{k}$. Assume for simplicity that $\mu=\mu_{k}$ is a single eigenvalue. The function $F(\mu)$ has a jump from negative infinity at $\mu=\mu_{k}-0$ to positive infinity at $\mu=\mu_{k}+0$, if the eigenfunction $u_{k}(x)$ at $\mu=\mu_{k}$ does not belong to constrained function space $X_{p}(\mathbb{R})$. Otherwise, i.e., if $u_{k}(x)$ lies in $X_{p}(\mathbb{R})$, the function $F(\mu)$ is continuous at $\mu=\mu_{k}$. The function $F(\mu)$ approaches -0 in the limit $\mu \rightarrow-\infty$ and it approaches a positive value in the limit $\mu \rightarrow 0$,

$$
F(0)=-\left\langle\Phi^{p}, \mathcal{H}^{-1} \Phi^{p}\right\rangle=\frac{1}{p-1}\left\langle\Phi^{p}, \Phi\right\rangle>0,
$$

where we have used the Parseval identity (2.2). The number of negative eigenvalues $\mu$ of operator $\mathcal{H}$ in $X_{p}(\mathbb{R})$ equals the number of zeros of the function $F(\mu)$ for $\mu<0$ and 
the number of eigenfunctions $u_{k}(x)$ of operator $\mathcal{H}$ that belongs to the space $X_{p}(\mathbb{R})$ for $\mu_{k}<0$. By continuity of the decreasing function $F(\mu)$ between $\mu \in\left[\mu_{k}, \mu_{k+1}\right]$ and by counting the jump discontinuity of $F(\mu)$ at $\mu=\mu_{k}$ [P04], we conclude that the number of negative eigenvalues $\mu$ of $\mathcal{H}$ in $X_{p}(\mathbb{R})$ equals $n(\mathcal{H})-1$.

Lemma 2.4. The spectrum of $(c+\mathcal{L})^{-1} \mathcal{H}$ in $X_{p}(\mathbb{R})$ has $n(\mathcal{H})-1$ negative eigenvalues $\lambda$.

Proof. By Sylvester's inertial theorem [M88, P04], the dimension of the negative space of the quadratic form $\langle U, \mathcal{H} U\rangle$ is invariant in any orthogonal basis of $X_{p}(\mathbb{R})$ that diagonalizes $\langle U, \mathcal{H} U\rangle$ with respect to a positive weighted inner product. One orthogonal basis for $X_{p}(\mathbb{R})$ is given by the eigenfunctions $\psi(x)$ of the constrained problem (2.7). The other orthogonal basis with respect to $(c+\mathcal{L})$ is defined by the eigenvalue problem (2.4). By invariance of the negative index of $\mathcal{H}$ in $X_{p}(\mathbb{R})$, we have $n(\mathcal{H})-1$ negative eigenvalues $\lambda$ in Problem II.

Lemma 2.5. The positive spectrum of $(c+\mathcal{L})^{-1} \mathcal{H}$ in $X_{p}(\mathbb{R})$ consists of infinitely many discrete eigenvalues $\lambda$ in the interval $0<\lambda<1$, accumulating to $\lambda \rightarrow 1^{-}$. If $\Phi^{p-1}(x) \geq 0$ for $x \in \mathbb{R}$, no eigenvalues $\lambda$ exists for $\lambda>1$. If there exists $x_{0} \in \mathbb{R}$ such that $\Phi^{p-1}\left(x_{0}\right)<0$, the spectrum of $(c+\mathcal{L})^{-1} \mathcal{H}$ also includes infinitely many discrete eigenvalues in the interval $1<\lambda \leq \lambda_{\max }$, accumulating to $\lambda \rightarrow 1^{+}$, where

$$
\lambda_{\max }<1+\frac{p}{c}\left|\min _{x \in \mathbb{R}} \Phi^{p-1}(x)\right|<\infty .
$$

Proof. Positive eigenvalues $\lambda$ can be estimated from (2.4) rewritten in the form

$$
(c+\mathcal{L}) U-\frac{p}{1-\lambda} \Phi^{p-1}(x) U=0 .
$$

Since $(c+\mathcal{L})$ is positive, no continuous spectrum of the problem $(2.12)$ exists. It was proved in [CM99] for a similar spectral problem that the spectrum of the problem (2.12) is discrete since $\operatorname{tr} M^{2}<\infty$, where $M=(c+\mathcal{L})^{-1 / 2} \Phi^{p-1}(c+\mathcal{L})^{-1 / 2}$ is a bounded operator. Since the spectrum of $\Phi^{p-1}(x)$ is infinite-dimensional, the spectrum of the bounded operator $M$ cannot be a finite rank [C01]. The potential term in (2.12) becomes singular in the limit $\lambda \rightarrow 1$ and therefore the point $\lambda=1$ is an accumulation point of the discrete eigenvalues. If $\Phi^{p-1}(x) \geq 0$ for any $x \in \mathbb{R}$, the positive part of $M$ and the spectrum of the problem (2.12) in the interval $0<\lambda<1$ are infinitedimensional, with eigenvalues accumulating to $\lambda \rightarrow 1^{-}$. In this case, no eigenvalues exist for $\lambda>1$, since

$$
\lambda=1-p \frac{\left\langle U, \Phi^{p-1} U\right\rangle}{\langle U,(c+\mathcal{L}) U\rangle}<1 .
$$

If $\Phi^{p-1}(x)$ changes sign on $x \in \mathbb{R}$, the negative part of $M$ and the spectrum of the problem (2.12) for $\lambda>1$ are infinite-dimensional, with eigenvalues accumulating to $\lambda \rightarrow 1^{+}[\mathrm{C} 01]$. Since $\langle U, \mathcal{L} U\rangle \geq 0$ and

$$
\left\langle U, \Phi^{p-1} U\right\rangle>-\left(\min _{x \in \mathbb{R}}\left|\Phi^{p-1}(x)\right|\right)\langle U, U\rangle,
$$

the largest positive eigenvalue $\lambda=\lambda_{\max }$ is bounded from above by (2.11). The spectrum of $(c+\mathcal{L})^{-1} \mathcal{H}$ is shown schematically on Figure 1.

COROLlary 2.6. The spectrum of $(c+\mathcal{L})^{-1} \mathcal{H}$ is located below $\lambda<1$ if and only if $p$ is odd or the bound state of the nonlinear problem (1.3) is nonnegative, $\Phi(x) \geq 0$ on $x \in \mathbb{R}$. 


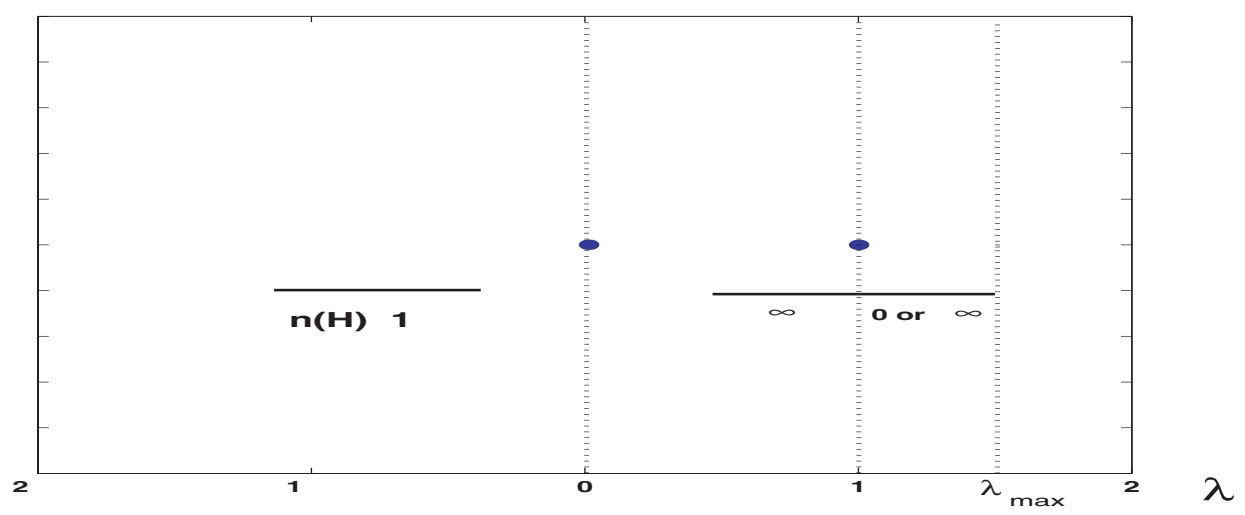

FIG. 1. Schematical representation of the spectrum of the operator $(c+\mathcal{L})^{-1} \mathcal{H}$.

Assumption 2.7. Either $\Phi^{p-1}(x) \geq 0$ on $x \in \mathbb{R}$ or $\lambda_{\max }<2$.

Our main theorem prescribes convergence or divergence of the iteration method (1.8)-(1.9).

THEOREM 2.8. Let $\hat{\Phi}(k)$ be a solution of the boundary-value problem (1.5) and Assumptions 1.1 and 2.1 be satisfied. The iteration method (1.8)-(1.9) converges to $\hat{\Phi}(k)$ in a small open neighborhood of $\hat{\Phi}(k)$ if (i) $1<\gamma<(p+1) /(p-1)$, (ii) $n(\mathcal{H})=1$, and (iii) Assumption 2.7 is met. The fastest rate of convergence occurs for $\gamma=\gamma_{*} \equiv$ $p /(p-1)$. If any of the three conditions are not met, the iteration method (1.8)-(1.9) diverges from $\hat{\Phi}(k)$.

3. Contraction of the iterative method near the fixed point. Our proof of Theorem 2.8 is based on the spectral analysis of the iteration operator (1.8)-(1.9), linearized at $\hat{\Phi}(k)$, and on the application of the contraction mapping principle for nonlinear operators [HP80].

Proposition 3.1. The iteration operator (1.8)-(1.9), linearized at $\hat{\Phi}(k)$, has a spectral radius smaller than one if and only if (i) $1<\gamma<(p+1) /(p-1)$, (ii) $n(\mathcal{H})=1$, and (iii) Assumption 2.7 is met.

Proof. Consider $\widehat{w}_{0}(k)=\widehat{u}_{0}(k)-\hat{\Phi}(k)$ be a small perturbation to $\hat{\Phi}(k)$, such that $\left\langle\Phi^{\prime}, w_{0}\right\rangle=0$. The sequence $\widehat{w}_{n}(k)=\widehat{u}_{n}(k)-\hat{\Phi}(k)$ is generated by the iteration operator (1.8), linearized at $\hat{\Phi}(k)$,

$$
\widehat{w}_{n+1}(k)=\gamma m_{n} \hat{\Phi}(k)+p \frac{\widehat{\Phi^{p-1}} * \widehat{w}_{n}(k)}{c+v(k)},
$$

where $*$ is the convolution operator and $m_{n}=M_{n}-1$. The correction $m_{n}$ is generated by the stabilizing factor (1.9), linearized at $\hat{\Phi}(k)$,

$$
m_{n}=(1-p) \frac{\int_{-\infty}^{\infty} \widehat{\Phi^{p}}(k) \widehat{w}_{n}(k) d k}{\int_{-\infty}^{\infty} \widehat{\Phi^{p}}(k) \hat{\Phi}(k) d k} .
$$

The correction term $w_{n}(x)$ can be decomposed explicitly as

$$
w_{n}=a_{n} \Phi(x)+q_{n}(x), \quad q_{n} \in X_{p}(\mathbb{R}),
$$

where $X_{p}(\mathbb{R})$ is defined by (2.6). It follows from (3.1) and (3.2) that $m_{n}=(1-p) a_{n}$ and $m_{n}$ solves the linear map

$$
m_{n+1}=[p-\gamma(p-1)] m_{n} .
$$


On the other hand, the correction term $q_{n}(x)$ solves the homogeneous part of the problem (3.1), which is equivalently rewritten on $x \in \mathbb{R}$ as

$$
q_{n+1}(x)=q_{n}(x)-(c+\mathcal{L})^{-1} \mathcal{H} q_{n}(x) .
$$

If $1<\gamma<(p+1) /(p-1)$, then $\lim _{n \rightarrow \infty} m_{n}=0$, such that $\lim _{n \rightarrow \infty} M_{n}=1$ for the stabilizing factor $M_{n}=1+m_{n}$. Therefore, the first term in the decomposition (3.3) vanishes as $n \rightarrow \infty$. The second term $q_{n}(x)$ may, however, remain finite or even grow with the number of iterations. We derive the conditions when $q_{n}(x)$ converges to zero as $n \rightarrow \infty$.

If $w_{0}(x)$ is orthogonal to $\Phi^{\prime}$, then $\left\langle\Phi^{\prime}, q_{0}\right\rangle=0$. It follows from (3.5) that $\left\langle\Phi^{\prime}, q_{n}\right\rangle=$ $0, \forall n$. We apply, therefore, the spectral decomposition of $X_{p}(\mathbb{R})$, described in Lemmas 2.4 and 2.5. The sequence $\left\{q_{n}(x)\right\}_{n=0}^{\infty}$ is decomposed through eigenfunctions $U_{k}(x)$ of the operator $(c+\mathcal{L})^{-1} \mathcal{H}$ as follows:

$$
q_{n}(x)=\sum_{k=1}^{n(\mathcal{H})-1} \alpha_{k}^{(n)} U_{k}(x)+\sum_{0<\lambda_{k}<1} \beta_{k}^{(n)} U_{k}(x)+\sum_{1<\lambda_{k} \leq \lambda_{\max }} \gamma_{k}^{(n)} U_{k}(x),
$$

where the first sum represents the finite-dimensional negative space of $X_{p}(\mathbb{R})$, the second sum represents the infinite-dimensional positive space of $X_{p}(\mathbb{R})$ for $0<\lambda<1$, and the third sum represents the infinite-dimensional positive space of $X_{p}(\mathbb{R})$ for $1<\lambda \leq \lambda_{\max }$, if the latter exists. The linear maps for coefficients of expansions are

$$
\begin{gathered}
\alpha_{k}^{(n+1)}=\left(1+\left|\lambda_{k}\right|\right) \alpha_{k}^{(n)}, \quad \lambda_{k}<0, \\
\beta_{k}^{(n+1)}=\left(1-\lambda_{k}\right) \beta_{k}^{(n)}, \quad 0<\lambda_{k}<1,
\end{gathered}
$$

and

$$
\gamma_{k}^{(n+1)}=\left(1-\lambda_{k}\right) \gamma_{k}^{(n)}, \quad 1<\lambda_{k} \leq \lambda_{\max }
$$

Iterations for coefficients $\alpha_{k}^{(n)}$ diverge for any $\lambda_{k}<0$. Iterations for coefficients $\beta_{k}^{(n)}$ converges for any $0<\lambda_{k}<1$. Iterations for coefficients $\gamma_{k}^{(n)}$ diverge for any $\lambda_{k} \geq 2$ and converge for $1<\lambda_{k}<2$. In the limit $n \rightarrow \infty$, the correction $q_{n}(x)$ uniformly converges to zero if the negative space of $X_{p}(\mathbb{R})$ is empty, i.e., $n(\mathcal{H})=1$, and the positive space of $X_{p}(\mathbb{R})$ is empty for $\lambda_{k} \geq 2$. The latter condition is satisfied under Assumption 2.7, i.e., when either the third sum in (3.6) is absent ( $p$ is odd or $\Phi(x) \geq 0$ on $x \in \mathbb{R}$ ) or $\lambda_{\max }<2$. We note that $\lambda_{\max }$ is bounded from above by (2.11).

Remark 3.2. In the proof of Proposition 3.1, we have assumed that $\left\langle\Phi^{\prime}, w_{0}\right\rangle=0$. If $w_{0}(x)$ does not satisfy the constraint, iterations of the linearized operator (3.1)(3.2) converge to the eigenfunction $\Phi^{\prime}(x)$ of the kernel of $\mathcal{H}$, which simply translates the bound state $\Phi(x)$ in $x$.

When the kernel of $\mathcal{H}$ has dimension greater than one, the corresponding eigenfunctions translate the bound state $\Phi(x)$ to some other solutions, which typically implies bifurcations of the bound states. It is expected that the iteration method (1.8)-(1.9) selects only one branch of solutions beyond the bifurcation, i.e., the other branches of solutions have the negative index $n(\mathcal{H})>1$. We eliminate the bifurcation cases by Assumption 2.1, which ensures that the kernel of $\mathcal{H}$ is one dimensional.

Remark 3.3. The rate of convergence of the stabilizing factor $M_{n}=1+m_{n}$ becomes superlinear if $\gamma=\gamma_{*}=p /(p-1)$, see (3.4). However, the corrections $q_{n}(x)$ 
still converge with the linear rate at $\gamma=\gamma_{*}$; see (3.5). Thus, the fastest but linear rate of convergence occurs at $\gamma=\gamma_{*}$. This conclusion confirms the Petviashvili's conjecture on the fastest rate of convergence [PP92].

Proposition 3.4. The iteration operator (1.8)-(1.9), linearized at the sequence $\left\{\hat{\phi}_{n}(k)\right\}_{n=0}^{\infty}$, is continuous in a small open neighborhood of $\hat{\Phi}(k)$.

Proof. Consider a difference $\delta \hat{u}_{n}(k)=\hat{u}_{n}(k)-\hat{\phi}_{n}(k)$ between any two sequences $\left\{\hat{u}_{n}(k)\right\}_{n=0}^{\infty}$ and $\left\{\hat{\phi}_{n}(k)\right\}_{n=0}^{\infty}$ generated by the iteration operator (1.8)-(1.9). The sequence $\delta \hat{u}_{n}(k)$ is defined by the iteration operator (1.8), linearized at $\hat{\phi}_{n}(k)$,

$$
\delta \hat{u}_{n+1}(k)=\gamma \frac{\delta M_{n}}{M_{n}} \hat{\phi}_{n+1}(k)+p M_{n}^{\gamma} \frac{\widehat{\phi_{n}^{p-1}} * \delta \hat{u}_{n}(k)}{c+v(k)},
$$

where $M_{n}=M_{n}\left[\hat{\phi}_{n}\right]$ and $\delta M_{n}=M_{n}\left[\hat{\phi}_{n}+\delta \hat{u}_{n}\right]-M_{n}\left[\hat{\phi}_{n}\right]$. The correction $\delta M_{n}$ is generated by the stabilizing factor (1.9), linearized at $\hat{\phi}_{n}(k)$,

$$
\delta M_{n}=\frac{2 \int_{-\infty}^{\infty}[c+v(k)] \hat{\phi}_{n}(k) \delta \hat{u}_{n}(k) d k-(1+p) M_{n} \int_{-\infty}^{\infty} \widehat{\phi_{n}^{p}}(k) \delta \hat{u}_{n}(k) d k}{\int_{-\infty}^{\infty} \widehat{\phi_{n}^{p}}(k) \hat{\phi}_{n}(k) d k}
$$

The linearized iteration operator (3.10)-(3.11) is continuous with respect to $\phi_{n} \in$ $X(\mathbb{R})$, where $X(\mathbb{R})$ is defined by $(1.6)$.

Proof of Theorem 2.8. The iteration method (1.8)-(1.9) represents a nonlinear operator $\hat{u}_{n+1}=\mathcal{A}\left(\hat{u}_{n}\right)$ in function space $X(\mathbb{R})$. The operator $\mathcal{A}\left(\hat{u}_{n}\right)$ has a continuous Frechet derivative $\mathcal{A}^{\prime}\left(\hat{u}_{n}\right)$ in small open neighborhood of $\hat{\Phi}$ in $X(\mathbb{R})$. Under the three conditions of Proposition 3.1, the spectral radius of $\mathcal{A}^{\prime}(\hat{\Phi})$ is smaller than one, i.e., $\left\|\mathcal{A}^{\prime}(\hat{\Phi})\right\|<1$. By continuity of the Frechet derivative, for any $\epsilon$ with $0<\epsilon<$ $1-\left\|\mathcal{A}^{\prime}(\hat{\Phi})\right\|$, there is a small open ball $S(\hat{\Phi}, \delta) \in X(\mathbb{R})$ centered at $\hat{\Phi}(k)$ with the radius $\delta=\delta(\epsilon)$, such that

$$
q=\sup _{\hat{u}_{n} \in S(\hat{\Phi}, \delta)}\left\|\mathcal{A}^{\prime}\left(\hat{u}_{n}\right)\right\|<1 .
$$

It follows from [HP80, Lemma 4.4.7] that

$$
\|\mathcal{A}(\hat{f})-\mathcal{A}(\hat{g})\| \leq q\|\hat{f}-\hat{g}\|
$$

for any $\hat{f}, \hat{g} \in S(\hat{\Phi}, \delta)$. Then, the contraction mapping theorem [HP80, Theorem 4.3.4] applies and the nonlinear operator $\mathcal{A}\left(\hat{u}_{n}\right)$ has a unique asymptotically stable fixed point for $\hat{u}_{n} \in S(\hat{\Phi}, \delta)$. Moreover, the asymptotic rate of convergence is determined by the Frechet derivative at $\hat{\Phi}$ as follows:

$$
\left\|\hat{u}_{n}-\hat{\Phi}\right\| \leq\left(\left\|\mathcal{A}^{\prime}(\hat{\Phi})\right\|+\epsilon\right)^{n}\left\|\hat{u}_{0}-\hat{\Phi}\right\| .
$$

See [HP80, Lemma 4.4.8] for further details.

4. Convergence of self-similar sequences. Here we derive conditions for convergence of a special sequence $\left\{x_{n} \hat{\Phi}(k)\right\}_{n=0}^{\infty}$, which is self-similar to $\hat{\Phi}(k)$ module to amplitude scaling. We also consider convergence of a general sequence in the small open neighborhood of $\left\{x_{n} \hat{\Phi}(k)\right\}_{n=0}^{\infty}$.

Proposition 4.1. Let $\hat{\Phi}(k)$ be a solution of the boundary-value problem (1.5) and Assumption 1.1 be satisfied. There exists a sequence $\left\{x_{n} \hat{\Phi}(k)\right\}_{n=0}^{\infty}$ in the iteration map (1.8)-(1.9), which converges to $\hat{\Phi}(k)$ for any $x_{0}>0$ if $1<\gamma<(p+1) /(p-1)$. 
Proof. Define $\hat{u}_{0}(k)=x_{0} \hat{\Phi}(k)$ for any $x_{0}>0$. Then, it follows from (1.5), (1.8), and (1.9) that $\hat{u}_{n}(k)=x_{n} \hat{\Phi}(k)$ for any $n \geq 0$, where $x_{n}$ is defined by the power iteration map

$$
x_{n+1}=M_{n}^{\gamma} x_{n}^{p}=x_{n}^{p-\gamma(p-1)},
$$

where $M_{n}=x_{n}^{1-p}$. The iteration map converges for $1<\gamma<(p+1) /(p-1)$ with the limit $\lim _{n \rightarrow \infty} x_{n}=1$. As a result, $\lim _{n \rightarrow \infty} \hat{u}_{n}(k)=\hat{\Phi}(k)$.

Remark 4.2. The rate of convergence of the power iteration map (4.1) is linear for $\gamma \neq \gamma_{*}$, where $\gamma_{*}=p /(p-1)$. When $\gamma=\gamma_{*}$, the convergence occurs in a single iteration: $\hat{u}_{1}(k)=\hat{\Phi}(k)$ for any $x_{0}>0$. The starting value $\hat{u}_{0}(k)$ is self-similar to the bound state $\hat{\Phi}(k)$ module to amplitude scaling. The special sequence $\left\{\hat{u}_{n}(k)\right\}_{n=0}^{\infty}$ exists in the iteration map (1.8)-(1.9) due to the power nonlinearity. The special sequence does not exist for general nonlinear functions.

Proposition 4.3. Let $\hat{\Phi}(k)$ be a solution of the boundary-value problem (1.5) and Assumptions 1.1 and 2.1 be satisfied. Let $\left\{x_{n} \hat{\Phi}(k)\right\}_{n=0}^{\infty}$ be a self-similar sequence, where $x_{n}$ is generated by the power iteration map (4.1) with any $x_{0}>0$. The iteration operator (1.8)-(1.9), linearized at $\left\{x_{n} \hat{\Phi}(k)\right\}_{n=0}^{\infty}$, has a spectral radius smaller than one if and only if (i) $1<\gamma<(p+1) /(p-1)$, (ii) $n(\mathcal{H})=1$, and (iii) Assumption 2.7 is met.

Proof. We use the linear map (3.10)-(3.11) with $\hat{\phi}_{n}(k)=x_{n} \hat{\Phi}(k)$, where $x_{n}$ solves the power iteration map (4.1). As a result, we find that $M_{n}=x_{n}^{1-p}$. The linear map (3.10)-(3.11) is then equivalent to the linear map (3.1)-(3.2) with the relations

$$
\hat{w}_{n}(k)=\frac{\delta \hat{u}_{n}(k)}{x_{n}}, \quad m_{n}=\frac{\delta M_{n}}{x_{n}^{p}} .
$$

Thus, Proposition 4.3 is equivalent to Proposition 3.1.

5. Examples in one dimension. Here we discuss two examples of the scalar wave equation (1.1), where the iteration method (1.8)-(1.9) can be used for finding stationary solutions such as solitary waves.

Example 5.1 (generalized Korteweg-de Vries (KdV) equations). A family of generalized $\mathrm{KdV}$ equations is defined for $\mathcal{L}=-\partial_{x}^{2}$, such that $v(k)=k^{2} \geq 0$ and $m=2$. The bound state solutions of the boundary-value problem (1.3) exist for $p>1$ in the analytical form (see, e.g., [PW92])

$$
\Phi(x)=\left[\sqrt{\frac{(p+1) c}{2}} \operatorname{sech}\left(\frac{p-1}{2} \sqrt{c} x\right)\right]^{\frac{2}{p-1}} .
$$

It follows from (5.1) that the bound state $\Phi(x)$ decays exponentially as

$$
\lim _{|x| \rightarrow \infty} \Phi(x) e^{\sqrt{c}|x|}=a_{\infty}, \quad a_{\infty}=[2(p+1) c]^{\frac{1}{p-1}} .
$$

The function $\Phi(x)$ belongs to $X(\mathbb{R})$ of Assumption 1.1. Since $\Phi(x) \geq 0$ on $x \in \mathbb{R}$, it also satisfies Assumption 2.7. The linearized operator $\mathcal{H}$ becomes a Schrödinger operator with a solvable potential,

$$
\mathcal{H}=c-\partial_{x}^{2}-\frac{p(p+1) c}{2} \operatorname{sech}^{2}\left(\frac{p-1}{2} \sqrt{c} x\right) .
$$


The Schrödinger operator (5.3) satisfies Assumption 2.1. Since $\mathcal{H} \Phi^{\prime}(x)=0$ and $\Phi(x)$ has no nodes on $x \in \mathbb{R}$, the Sturm oscillation theorem predicts only one negative eigenvalue of $\mathcal{H}$, i.e., $n(\mathcal{H})=1$. As a result, Theorem 2.8 applies and the iteration method (1.8)-(1.9) converges to the bound state $\hat{\Phi}(k)$ in the generalized KdV equation for any value of $p>1$ if $1<\gamma<(p+1) /(p-1)$.

Remark 5.2. In accordance with Theorem 2.2, the bound state $\Phi(x)$ is weakly spectrally stable with respect to the time evolution problem for $p<5$ and spectrally unstable for $p \geq 5$ (see also [BSS87, PW92]). On the other hand, the iteration method (1.8)-(1.9) converges for any $p>1$, irrelevantly to the stability of bound states in the time evolution problem. For instance, the interval of convergence with $p=5$ is $1<\gamma<3 / 2$ and the interval shrinks to zero when $p \rightarrow \infty$.

Example 5.3 (generalized Benjamin-Ono (BO) equations). A family of generalized $\mathrm{BO}$ equations is defined for $\mathcal{L}=-\partial_{x} H$, where $H(u)$ is the Hilbert transform of $u(x)$,

$$
H(u)=\frac{1}{\pi} \wp \int_{-\infty}^{\infty} \frac{u(z) d z}{z-x},
$$

and the symbol $\wp$ denotes the principal value of the integral. In this case, $v(k)=$ $|k| \geq 0$ and $m=1$. The bound state solutions of the nonlinear problem (1.3) are unknown in the analytical form except for the case $p=2$, when

$$
\Phi(x)=\frac{2 c}{1+c^{2} x^{2}} .
$$

Using the asymptotic representation for $\Phi(x) \in L^{1}(\mathbb{R})$,

$$
H(\Phi)=-\frac{1}{\pi x} \int_{-\infty}^{\infty} \Phi(z) d z+\mathrm{O}\left(\frac{1}{x^{2}}\right)
$$

and the balance of inverse powers of $x$ in the problem (1.3), we derive the algebraic decay of $\Phi(x)$ at infinity,

$$
\lim _{|x| \rightarrow \infty} x^{2} \Phi(x)=a_{-2}, \quad a_{-2}=\frac{1}{\pi c} \int_{-\infty}^{\infty} \Phi(x) d x .
$$

The function $\Phi(x)$ has sufficient decay at infinity to belong to $X(\mathbb{R})$ of Assumption 1.1 , if it exists for $p>1$. Since $\Phi(x) \geq 0$ on $x \in \mathbb{R}$ as follows from our numerical approximations (see Figure 2), Assumption 2.7 is satisfied. The linearized operator $\mathcal{H}$ becomes a nonlocal operator,

$$
\mathcal{H}=c-\partial_{x} H-p \Phi^{p-1}(x) .
$$

It was proved in [CK80] for $p=2$ that the nonlocal linearized operator (5.7) satisfies Assumption 2.1 and has only one negative eigenvalue, i.e., $n(\mathcal{H})=1$. As a result, Theorem 2.8 states that the iteration method (1.8)-(1.9) converges to the bound state $\hat{\Phi}(k)$ for the case $p=2$ if $1<\gamma<3$.

We have computed the bound states $\Phi(x)$ for $p=2,3,4,5$ from the iteration method (1.8)-(1.9) starting with the Gaussian approximation $u_{0}(x)=\exp \left(-x^{2}\right)$ for $c=1$ (see also [AS87]). The numerical approximations are plotted on Figure 2, where dots for $p=2$ show the exact values from (5.5). Figure 3 shows convergence of the stabilizing factor $M_{n}$ in the iteration method (1.8)-(1.9) with $p=2$, for three 


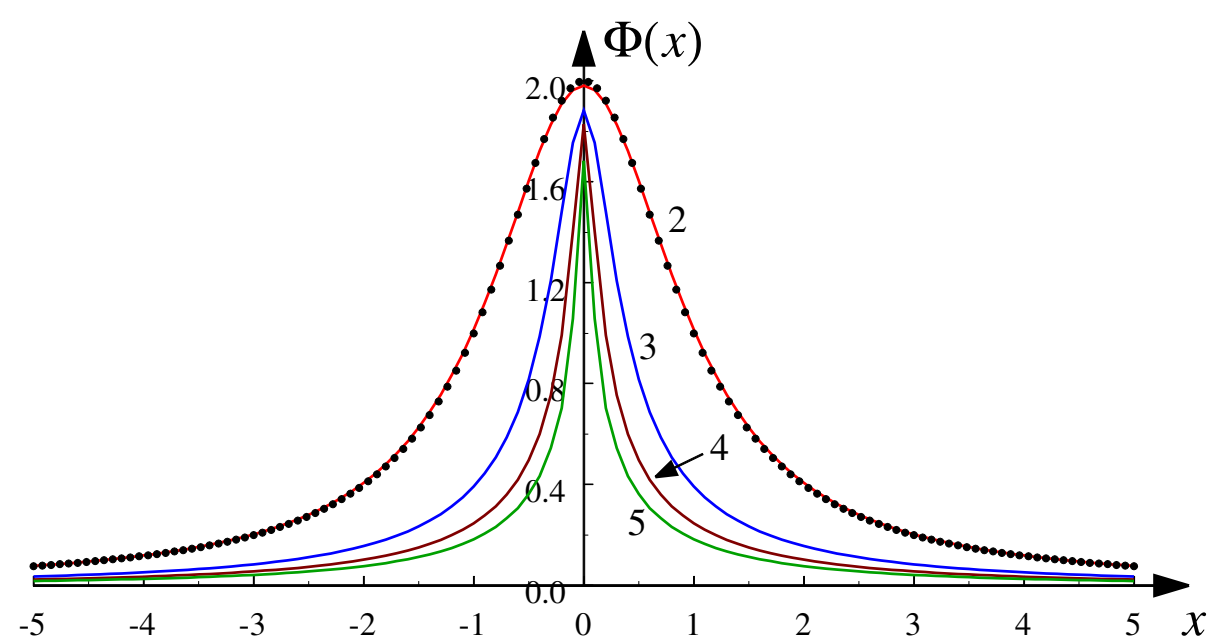

FIG. 2. Numerical approximations of the bound states $\Phi(x)$ of the generalized BO equation for $p=2,3,4,5$. Dots on curve 2 show exact values from the analytical solution (5.5).

different values of $\gamma: \gamma=2$ (dots), when the rate of convergence is the fastest; $\gamma=1.1$ (triangles), near the left boundary of the convergence interval; and $\gamma=2.9$ (crosses), near the right boundary of the convergence interval. We conclude that the iteration method (1.8)-(1.9) converges to the bound state of the generalized BO equation for $p=2,3,4,5$ if $1<\gamma<(p+1) /(p-1)$. Moreover, numerical computations show convergence of the method to a positive-definite bound state $\Phi(x)$ for any $p>1$, including noninteger values of $p$.

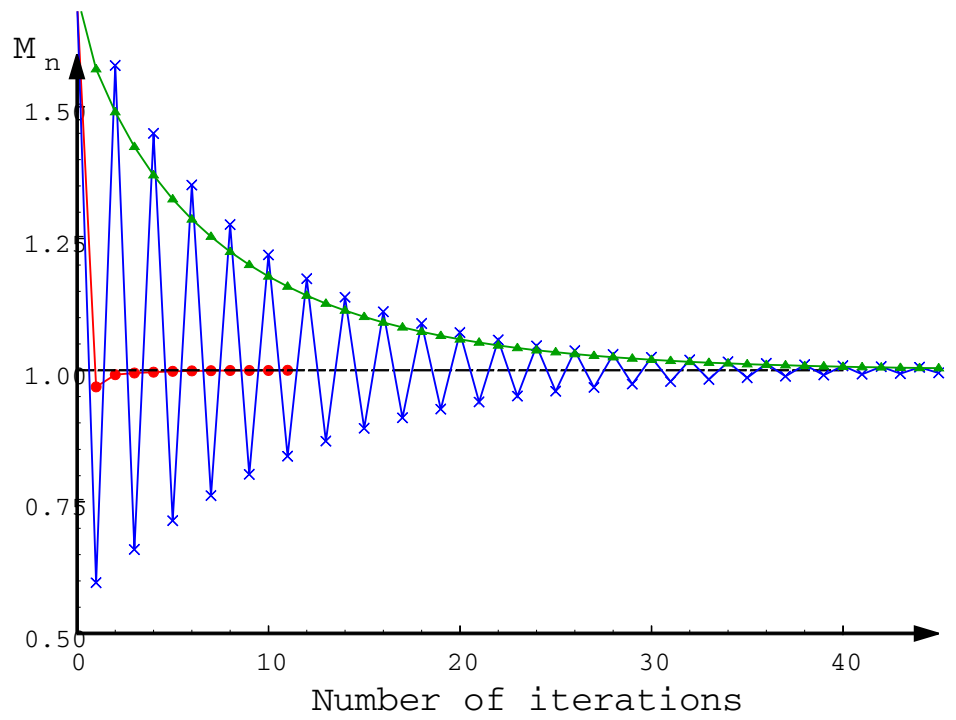

FIG. 3. Stabilizing factor $M_{n}$ versus $n$ for the iteration method (1.8)-(1.9) with $p=2$ for $\gamma=2$ (dots), $\gamma=1.1$ (triangles), and $\gamma=2.9$ (crosses). 
Remark 5.4. The bound state $\Phi(x)$ is spectrally stable with respect to the time evolution problem for $p=2$ and spectrally unstable for $p \geq 3$ [BSS87, CK80]. Under assumption that $n(\mathcal{H})=1$ and $\Phi(x) \geq 0$ on $x \in \mathbb{R}$ for any $p>1$, the iteration method (1.8)-(1.9) converges to the bound state for any $p>1$, irrelevantly to the stability of bound states in the time evolution problem. Therefore, the method becomes useful for numerical approximations of the bound states in the generalized BO equation, when exact analytical expressions are not available. In fact, the iteration method (1.8)-(1.9) was successfully used for numerical approximations of soliton solutions in the generalized $\mathrm{BO}$ and $\mathrm{KdV}$ equations in [AS87]. Another numerical method is developed with the help of Newton iteration algorithms but the Newton iterations have convergence problems as pointed out in [BK03]. We notice that Petviashvili's iteration method (1.8)-(1.9) is not sensitive to the choice of a starting function, which is its great advantage compared to the Newton's iteration method.

6. Examples in two dimensions. We finish the article with generalizations of the iteration method (1.8)-(1.9) for the scalar wave equation in space of two dimensions,

$$
u_{t}-(\mathcal{L} u)_{x}+p u^{p-1} u_{x}=0
$$

where $u: \mathbb{R}^{2} \times \mathbb{R}_{+} \mapsto \mathbb{R}, p>1$, and $\mathcal{L}$ is a linear self-adjoint nonnegative pseudodifferential operator in $x$ and $y$ with constant coefficients. If the Fourier transform (1.4) is replaced by the double Fourier transform in $L^{2}\left(\mathbb{R}^{2}\right)$, the iteration method (1.8)-(1.9) can be applied to the scalar wave equation (6.1) in two dimensions. The only modification is required for Assumption 2.1, since the kernel of $\mathcal{H}=c+\mathcal{L}-$ $p \Phi^{p-1}(x, y)$ has at least two eigenfunctions $\partial_{x} \Phi(x, y)$ and $\partial_{y} \Phi(x, y)$.

Assumption 6.1. The spectrum of $\mathcal{H}$ in $L^{2}\left(\mathbb{R}^{2}\right)$ consists of eigenvalues $\mu$ of the discrete spectrum for $\mu<c$ and the continuous spectrum for $\mu \geq c$. The null-space of $\mathcal{H}$ is two dimensional with the eigenfunctions $\partial_{x} \Phi(x, y)$ and $\partial_{y} \Phi(x, y)$. The negative space of $\mathcal{H}$ has dimension $n(\mathcal{H}) \geq 1$.

With this modification, we formulate the results of sections 2 and 3 as the following theorem.

THEOREM 6.2. Let $\hat{\Phi}(k)$ be a solution of the boundary-value problem (1.5) and Assumptions 1.1 and 6.1 be satisfied. The iteration method (1.8)-(1.9) converges to $\hat{\Phi}(k)$ in a small open neighborhood of $\hat{\Phi}(k)$ if (i) $1<\gamma<(p+1) /(p-1)$, (ii) $n(\mathcal{H})=1$, and (iii) Assumption 2.7 is met. The fastest rate of convergence occurs for $\gamma=\gamma_{*} \equiv$ $p /(p-1)$. If any of the three conditions are not met, the iteration method (1.8)-(1.9) diverges from $\hat{\Phi}(k)$.

Here we discuss three examples of the scalar wave equation (6.1) in two dimensions, where the iteration method (1.8)-(1.9) can be used for finding stationary solutions such as solitary waves.

Example 6.3 (generalized Zakharov-Kuznetsov (ZK) equations). The generalized $\mathrm{KdV}$ equations of Example 5.1 are extended to the two-dimensional ZK equations, when $\mathcal{L}$ is an isotropic operator,

$$
\mathcal{L}=-\left(\partial_{x}^{2}+\partial_{y}^{2}\right)
$$

such that $v(k)=k_{x}^{2}+k_{y}^{2} \geq 0$. The bound state $u=\Phi(x-c t, y)$ satisfies the nonlinear problem

$$
c \Phi-\Delta \Phi=\Phi^{p} .
$$


Existence and uniqueness of positive solutions of the nonlinear elliptic problem (6.3) was proved for any $p>1$ [GNN81, K89] such that $\Phi(x, y)=\Phi(r)$ is radially symmetric, where $r=\sqrt{x^{2}+y^{2}}$, and satisfies the limiting decay

$$
\lim _{r \rightarrow \infty} e^{\sqrt{c} r} r^{1 / 2} \Phi(r)=a_{\infty}>0 .
$$

The positive solutions $\Phi(r)$ satisfy Assumptions 1.1 and 2.7. The linearized operator $\mathcal{H}$ becomes the Schrödinger operator with the radially symmetric potential

$$
\mathcal{H}=c-\partial_{x}^{2}-\partial_{y}^{2}+p \Phi^{p-1}(r) .
$$

Assumption 6.1 is satisfied for the Schrödinger operator (6.5) and the negative index of $\mathcal{H}$ for the positive ground state $\Phi(r)$ is one, i.e., $n(\mathcal{H})=1$ [S9, p. 63]. Therefore, iterations of the numerical method (1.8)-(1.9) converge for $1<\gamma<(p+1) /(p-1)$, according to Theorem 6.2 for any $p>1$. This result justifies the use of the iteration method (1.8)-(1.9) for numerical approximation of bound states of the generalized ZK equations.

Example 6.4 (generalized Kadomtsev-Petviashvili (KP) equations). The generalized KdV equations of Example 5.1 are extended to the two-dimensional KP equations, when $\mathcal{L}$ is an anisotropic operator,

$$
\mathcal{L}=-\partial_{x}^{2}+\partial_{x}^{-2} \partial_{y}^{2}
$$

such that $v(k)=k_{x}^{2}+k_{x}^{-2} k_{y}^{2} \geq 0$. The linear operator $\mathcal{L}$ in (6.6) corresponds to the KPI equation with two-dimensional solitons, called lumps. The nonlocal $\partial_{x}^{-1}$ operator is well posed subject to the constraint on $u(x, y, t)$

$$
\int_{-\infty}^{\infty} u(x, y, t) d x=0
$$

The bound state $u=\Phi(x-c t, y)$ satisfies the nonlinear problem

$$
c \Phi-\Phi_{x x}+\partial_{x}^{-2} \Phi_{y y}=\Phi^{p} .
$$

The exact analytical solution for $\Phi(x, y)$ exists for $p=2$ [MZ77],

$$
\Phi(x, y)=12 c \frac{3+c^{2} y^{2}-c x^{2}}{\left(3+c^{2} y^{2}+c x^{2}\right)^{2}} .
$$

The bound state $\Phi(x, y)$ is sign-indefinite due to the constraint (6.7). Existence of sign-indefinite bound states in the nonlinear problem (6.8) was proved for $p=3,4$ by using constrained minimization [BS97]. It was also shown that the solution exists only for $p<5$ and $p=p_{1} / p_{2}$, where $p_{1}$ is any even integer and $p_{2}$ is any odd integer [LW97]. Bound states $\Phi(x, y)$ satisfy Assumption 1.1.

It can be shown with the Riemann-Hilbert inverse scattering method [PS00] that the spectrum of $\mathcal{H}$ for $p=2$ satisfies Assumption 6.1 with $n(\mathcal{H})=1$. Since the bound states $\Phi(x, y)$ are nonpositive, they satisfy Assumption 2.7 only if $\lambda_{\max }<2$. It follows from (6.9) for $p=2$ that 


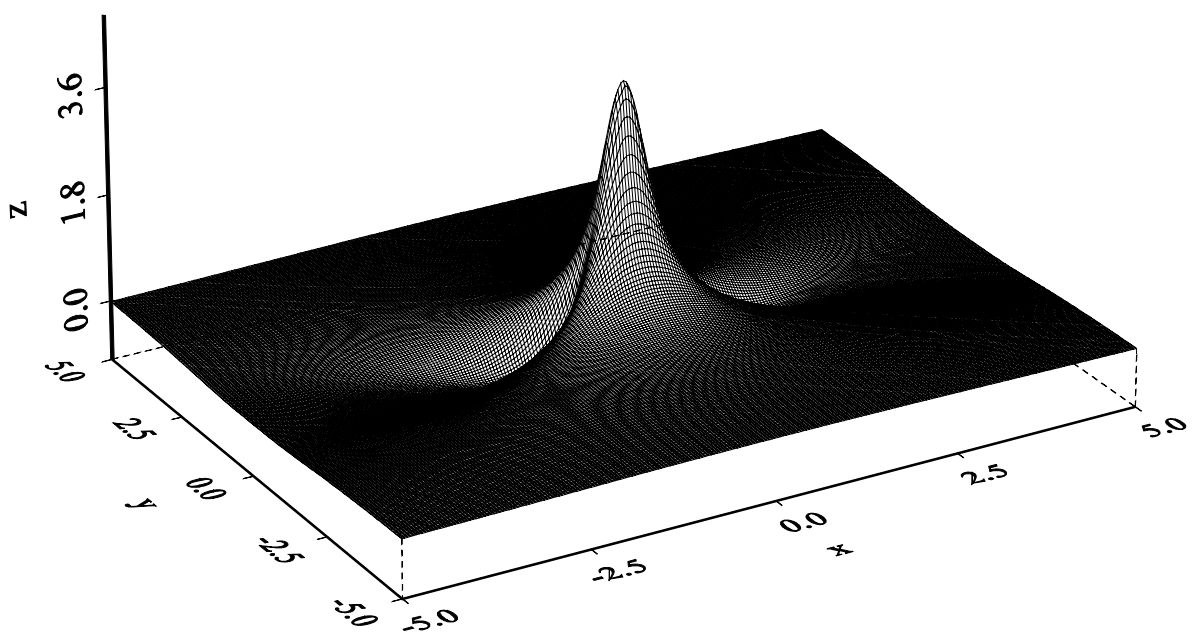

FIG. 4. A numerical approximation of the bound state $\Phi(x, y)$ of the generalized KPI equation with $p=3$.

$$
\min _{(x, y) \in \mathbb{R}^{2}} \Phi(x, y)=\Phi\left( \pm \frac{3}{\sqrt{c}}, 0\right)=-\frac{c}{2}
$$

Therefore, the upper bound (2.11) applies with $\lambda_{\max }<1+1=2$, i.e., Assumption 2.7 is also satisfied. Theorem 6.2 states that the iteration method (1.8)(1.9) converges to $\Phi(x, y)$ for $p=2$ if $1<\gamma<3$. This analysis justifies the use of the numerical iteration method (1.8)-(1.9), proposed originally by Petviashvili $[\mathrm{P} 76]$.

We have computed the bound states $\Phi(x, y)$ for $p=2,3,4$ from the iteration method (1.8)-(1.9) starting with the lump solution (6.9) with $c=1$. The constraint (6.7) is built into the algorithm as zero Fourier mode with $k_{x}=0$. The final solution $\Phi(x, y)$ is shown on Figure 4 for $p=3$ (see also [AS87]). Cross-sections $\Phi(x, 0)$ and $\Phi(0, y)$ are shown on Figure $5(\mathrm{a}, \mathrm{b})$ for $p=2,3,4$, where dots for $p=2$ show exact values from (6.9). Figure 6 shows convergence of the stabilizing factor $M_{n}$ in the iteration method (1.8)-(1.9) with the fastest rate $\gamma=p /(p-1)$ for $p=2,3,4$. We conclude that the iteration method (1.8)-(1.9) converges to the bound state of the generalized KP equation for $p=2,3,4$ if $1<\gamma<(p+1) /(p-1)$.

Remark 6.5. Nonpositive bound states of the generalized KP equations may consist of several individual lumps. Multilump solutions of the KPI equation with $p=2$ were discovered both numerically [AS85] and analytically [PS93]. However, a discrepancy occurs between the numerical and analytical solutions for a double-lump; the analytical solution is unique for the double-lump [PS93], while the numerical solution represents a continuous family with a free parameter of the distance between the two lumps [AS85]. This discrepancy is likely to be explained by low accuracy of the numerical procedure in [AS85], i.e., low resolution of the numerical mesh and small grid size. Since the negative index of $\mathcal{H}$ for multilump solutions typically exceeds one, the iteration method (1.8)-(1.9) must diverge in the neighborhood of multilump solutions, according to Theorem 6.2. Numerical approximations obtained in [AS85] are likely supported by the truncation of the domain on $\mathbb{R}^{2}$ and the discretization 

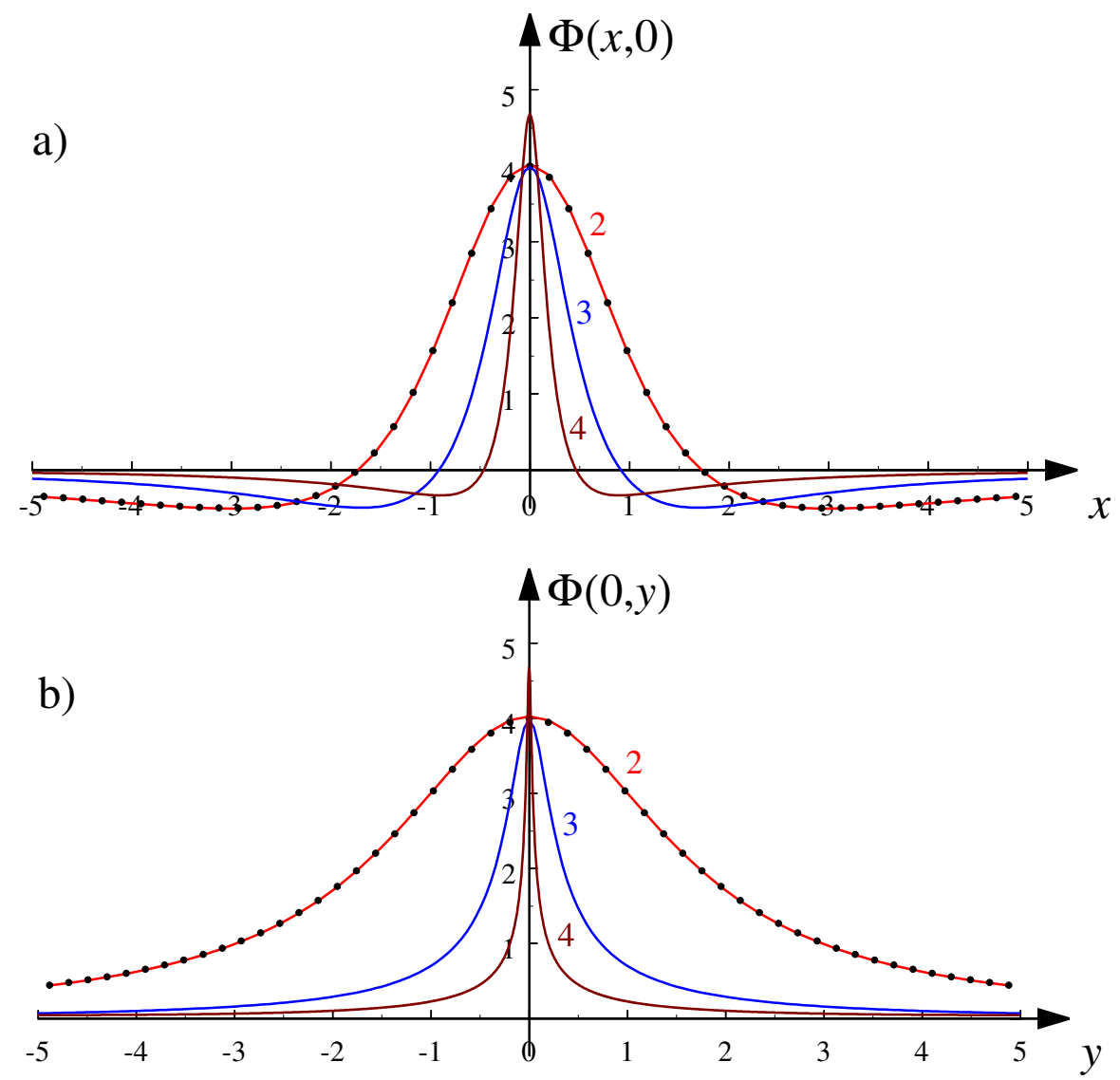

FIG. 5. Cross sections $\Phi(x, 0)$ and $\Phi(0, y)$ of numerical approximations of the bound states $\Phi(x, y)$ of the generalized KPI equation with $p=2,3,4$. Dots on curves 2 show exact values from the analytical solution (6.9).

of the numerical grid $(x, y)$. This example shows a danger of the direct use of the iteration method (1.8)-(1.9) without analysis of the three conditions of convergence in Theorems 2.8 and 6.2 .

Example 6.6 (generalized Klein-Gordon (KG) equations). Our last example shows that the iteration method (1.8)-(1.9) can be used for other nonlinear problems, such as the generalized KG equation,

$$
u_{t t}-c_{0}^{2}\left(u_{x x}+u_{y y}\right)+u=u^{p} .
$$

Travelling wave solutions of (6.10) are of the form $u(x, y, t)=\Phi(x-c t, y)$, where $\Phi(x, y)$ satisfies the boundary-value problem

$$
\Phi-\left(c_{0}^{2}-c^{2}\right) \Phi_{x x}-c_{0}^{2} \Phi_{y y}=\Phi^{p} .
$$

If $|c|<c_{0}$, the boundary-value problem (6.11) can be reduced to the form (6.3) of Example 6.3 with a simple rescaling of variables $x$ and $y$. 


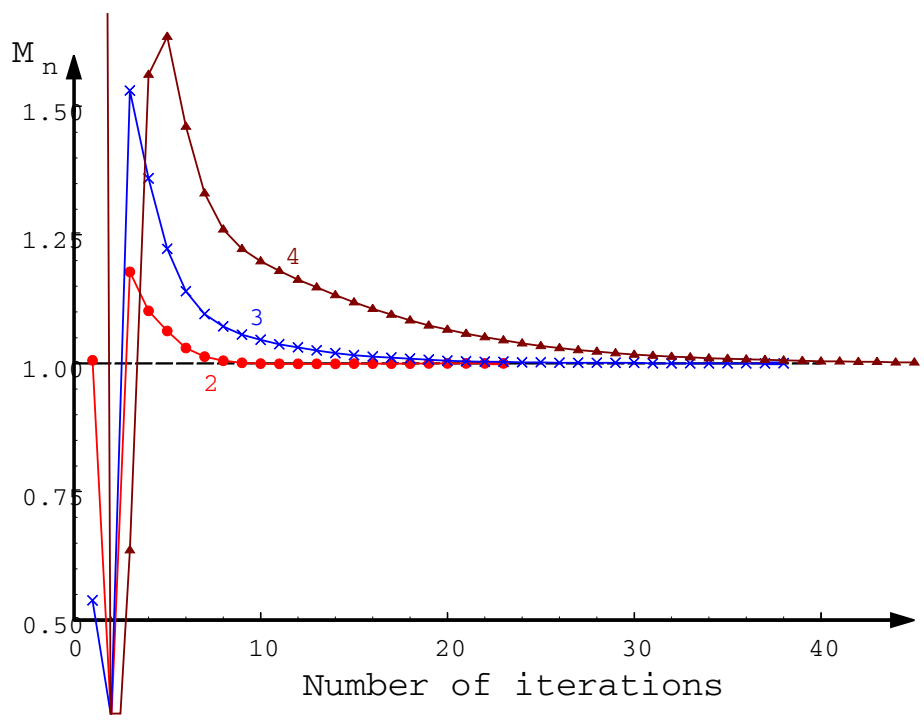

FIG. 6. Stabilizing factor $M_{n}$ versus $n$ in the iteration method (1.8)-(1.9) with the fastest rate $\gamma=p /(p-1)$ for $p=2,3,4$.

Acknowledgments. The authors are thankful to H. Kalisch and A. N. Notik for collaboration on the early stage of the work. One of the authors (D. P.) appreciates useful discussions with R. Gadyl'shin, A. Pushnitski, A. Scheel, and V. Vougalter.

\section{REFERENCES}

[AS85] L. A. Abramyan and Yu. A. Stepanyants, Two-dimensional multisolitons: stationary solutions of the Kadomtsev-Petviashvili equation, Radiophysics and Quantum Electronics, 28 (1985), pp. 20-26.

[AS87] L. A. Abramyan And Yu. A. Stepanyants, Structure of two-dimensional solitons in the context of a generalized Kadomtsev-Petviashvili equation, Radiophysics and Quantum Electronics, 30 (1987), pp. 861-865.

[BS87] M. Sh. Birman and M. Z. Solomyak, Spectral Theory of Self-Adjoint Operators in Hilbert Space, Reidel, Dordrecht, The Netherlands, 1987.

[BK03] J. Bona AND H. Kalisch, Singularity Formation in the Generalized Benjamin-Ono Equation, preprint, 2003.

[BSS87] J. L. Bona, P. E. Souganidis, And W. A. Strauss, Stability and instability of solitary waves of Korteweg-de Vries type, Proc. Roy. Soc. London Ser. A, 411 (1987), pp. 395412.

[BS97] A. De Bound and J. C. SAut, Solitary waves of generalized Kadomtsev-Petviashvili equations, Ann. Inst. H. Poincare, Anal. Non Lineaire, 14 (1997), pp. 211-236.

[CK80] H. H. CHEN AND D. J. KAUP, Linear stability of internal wave solitons in a deep stratified fluid, Phys. Fluids, 23 (1980), pp. 235-238.

[C01] A. Constantin, On the scattering problem for the Camassa-Holm equation, Proc. R. Soc. Lond. Ser. A Mat. Phys. Eng. Sci. 457 (2001), pp. 953-970.

[CM99] A. Constantin And H. P. MCKean, A shallow water equation on the circle, Comm. Pure Appl. Math., 52 (1999), pp. 949-982.

[GNN81] B. GidAs, W. M. NI, AND L. NiRENBERG, Symmetry of positive solutions of nonlinear elliptic equations in $\mathbb{R}^{N}$, in Mathematical Analysis and Applications, Adv. in Math. Suppl. Studies 7A, L. Nachbin, ed., Academic Press, New York, 1981, pp. 369-402.

[HP80] V. Hutson And J. S. Pym, Applications of Functional Analysis and Operator Theory, Academic Press, London, 1980. 
[K89] M. K. Kwong, Uniqueness of positive solutions of $\Delta u-u+u^{p}=0$ in $\mathbb{R}^{N}$, Arch. Rational Mech. Anal., 105 (1989), pp. 243-266.

[LW97] Y. LIU AND X. P. WANG, Nonlinear stability of solitary waves of a generalized Kadomtsev-Petviashvili equation, Comm. Math. Phys., 183 (1997), pp. 253-266.

[M88] J. H. MADDOCKS, Restricted quadratic forms, inertia theorems, and the Schur complement, Linear Algebra Appl., 108 (1988), pp. 1-36.

[MZ77] S. V. Manakov, V. E. Zakharov, L. A. BordaG, A. R. Its, and V. B. Matveev, Twodimensional solitons of the Kadomtsev-Petviashvili equation and their interaction, Phys. Lett. A, 63 (1977), pp. 205-206.

[PW92] R. L. Pego And M. I. Weinstein, Eigenvalues, and instabilities of solitary waves, Phil. Trans. Roy. Soc. London Ser. A, 340 (1992), pp. 47-94.

[P04] D. Pelinovsky, Inertia law for spectral stability of solitary waves in coupled nonlinear Schrödinger equations, preprint, Proc. Roy. Soc. Lond. Ser. A, (2004).

[PS93] D. E. Pelinovsky and Yu. A. Stepanyants, New multisoliton solutions of the Kadomtsev-Petviashvili equations, JETP Lett., 57 (1993), pp. 24-28.

[PS00] D. E. Pelinovsky AND C. SulEm, Eigenfunctions and eigenvalues for a scalar RiemannHilbert problem associated to inverse scattering, Comm. Math. Phys., 208 (2000), pp. $713-760$.

[P76] V. I. Petviashvili, Equation of an extraordinary soliton, Plasma Physics, 2 (1976), p. 469.

[PP92] V. I. Petviashvili and O. V. Pokhotelov, Solitary Waves in Plasmas and in the Atmosphere, Gordon and Breach, Philadelphia, 1992.

[S9] W. A. Strauss, Nonlinear Wave Equations, CBMS Reg. Conf. Ser. Math. 73, AMS, Providence, RI, 1989. 